\title{
On Hereditary Helly Classes of Graphs
}

\author{
Marina Groshaus If and Jayme L Szwarcfiter" \\ ${ }^{1}$ Universidad de Buenos Aires, Fac. de Ciencias Exactas y Naturales, Dep. de Computación, groshaus@dc.uba.ar \\ ${ }^{2}$ Universidade Federal do Rio de Janeiro Instituto de Matemática, NCE and COPPE, jayme@nce.ufrj.br
}

received february 20, 2006, revised november 24, 2007, accepted october 14, 2007.

\begin{abstract}
In graph theory, the Helly property has been applied to families of sets, such as cliques, disks, bicliques, and neighbourhoods, leading to the classes of clique-Helly, disk-Helly, biclique-Helly, neighbourhood-Helly graphs, respectively. A natural question is to determine for which graphs the corresponding Helly property holds, for every induced subgraph. This leads to the corresponding classes of hereditary clique-Helly, hereditary disk-Helly, hereditary biclique-Helly and hereditary neighbourhood-Helly graphs. In this paper, we describe characterizations in terms of families of forbidden subgraphs, for the classes of hereditary biclique-Helly and hereditary neighbourhood-Helly graphs. We consider both open and closed neighbourhoods. The forbidden subgraphs are all of fixed size, implying polynomial time recognition for these classes.
\end{abstract}

Keywords: Algorithms, bicliques, Helly property, graph classes

\section{Introduction}

In the scope of graph theory, the Helly property has been applied to families of sets as cliques, e.g. [6], [10], [12], [14], [19], bicliques [11], neighbourhoods [5] and disks [1], [3], [4]. The corresponding graphs are the clique-Helly, biclique-Helly, neighbourhood-Helly and disk-Helly graphs, respectively. Bicliques, in general, have been considered in some different contexts, e.g. [15, 16, 17, 20].

Besides the interest of examining bicliques in the scope of the Helly property, these graphs should be of interest in the study of retracts [13]. In fact, retracts of bipartite graphs are related to neighbourhoodHelly graphs and the latter are related to biclique-Helly graphs in some different aspects. See [2]. We also mention that some optimization problems, as the edge modification problem, have been already studied for the class of biclique-Helly graphs [8].

None of the above classes are closed under induced subgraphs. So, a question would be to characterize the graphs for which the Helly property is preserved for every induced subgraph. It leads to the hereditary classes of clique-Helly, biclique-Helly, neighbourhood-Helly and disk-Helly graphs. Hereditary clique-Helly graphs have been characterized in [18], while [9] (see [7]) contains a characterization of hereditary disk-Helly graphs. In this work, we describe forbidden subgraph characterizations for the

\footnotetext{
$\dagger$ Partially supported by UBACyT Grants X036, X184, X127, PICT ANPCyT Grant 11-09112 and PID Conicet Grant, Argentina.

¥Partially supported by the Conselho Nacional de Desenvolvimento Científico e Tecnológico, CNPq, and Fundação de Amparo à Pesquisa do Estado do Rio de Janeiro, FAPERJ, Brasil
}

1365-8050 @ 2008 Discrete Mathematics and Theoretical Computer Science (DMTCS), Nancy, France 


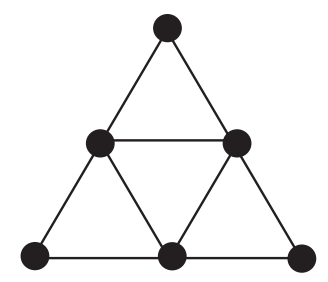

Fig. 1: The Hajós graph

classes of hereditary biclique-Helly and hereditary neighbourhood-Helly graphs. Both open and closed neighbourhoods are considered. All graphs in these forbidden families are of fixed size. In fact they have at most 8 vertices. Consequently, the characterizations imply polynomial time recognition for all the considered classes.

Denote by $G$ a finite simple graph, with vertex set $V(G)$ and edge set $E(G)$. For $v_{i}, v_{j} \in V(G)$, write $v_{i} v_{j} \in E(G)$ to denote an edge with ends $v_{i}$ and $v_{j}$. A complete set is a subset $V^{\prime} \subseteq V(G)$ formed by pairwise adjacent vertices while in an independent set $V^{\prime} \subseteq V(G)$ every pair of its vertices is not adjacent. Denote by $\alpha(G)$ the size of the largest independent set of $G$. A clique is a maximal complete set. A biclique is a subset $B \subseteq V(G)$ inducing a maximal complete bipartite graph in $G$, having at least one edge. Denote by $N\left(v_{i}\right)=\left\{v_{j} \in V(G) \mid v_{i} v_{j} \in E(G)\right\}$, and $N\left[v_{i}\right]=N\left(v_{i}\right) \cup\left\{v_{i}\right\}$, the open and closed neighbourhoods of $G$, respectively. For each non-negative integer $k$ and $v_{i} \in V(G)$, a disk $D_{k}\left(v_{i}\right)$ of $v_{i}$ is the set of vertices lying at distance at most $k$ from $v_{i}$. For $V^{\prime} \subseteq V(G)$, denote by $G\left[V^{\prime}\right]$ the subgraph of $G$ induced by $V^{\prime}$. Let $\mathcal{B}$ be a set of bicliques of $G$. Represent by $G_{\mathcal{B}}$ the biclique subgraph of $\mathcal{B}$, that is, the subgraph of $G$ formed exactly by the vertices and edges of $\mathcal{B}$.

Let $\mathcal{F}$ be a family of subsets of some set. Say that $\mathcal{F}$ is intersecting when the subsets of $\mathcal{F}$ pairwise intersect. On the other hand, when every intersecting subfamily of $\mathcal{F}$ has a common element then $\mathcal{F}$ is a Helly family. A graph $G$ is clique-Helly when its family of cliques is Helly. Similarly, $G$ is biclique-Helly (open neighbourhood-Helly, closed neighbourhood-Helly) when its family of bicliques (open neighbourhoods, closed neighbourhoods) is Helly. Also, say that $G$ is disk-Helly when the family of disks $\left\{D_{k}\left(v_{i}\right) \mid v_{i} \in V(G)\right.$ and $\left.1 \leq k<|V(G)|\right\}$ is Helly.

Finally, $G$ is hereditary clique-Helly when every of its induced subgraphs is clique-Helly. Similarly, define hereditary biclique-Helly, hereditary open neighbourhood-Helly, hereditary closed neighbourhoodHelly and hereditary disk-Helly graphs.

Hereditary clique-Helly graphs have been characterized as follows.

Theorem 1.1 [18]: A graph $G$ is hereditary clique-Helly if and only if it does not contain as induced subgraphs the Hajós graph and any graph obtained from the latter by inserting some edges joining its vertices of degree 2 .

The following is a characterization for hereditary disk-Helly graphs.

Theorem 1.2 [9]: A graph $G$ is hereditary disk-Helly if and only if it is chordal and does not contain the Hajós graph as an induced subgraph.

In Section 2, we describe a characterization for hereditary biclique-Helly graphs while the proposed characterizations for hereditary open and closed neighborhood-Helly graphs are in Section 3 . Relations among these classes are formulated in Section 4 

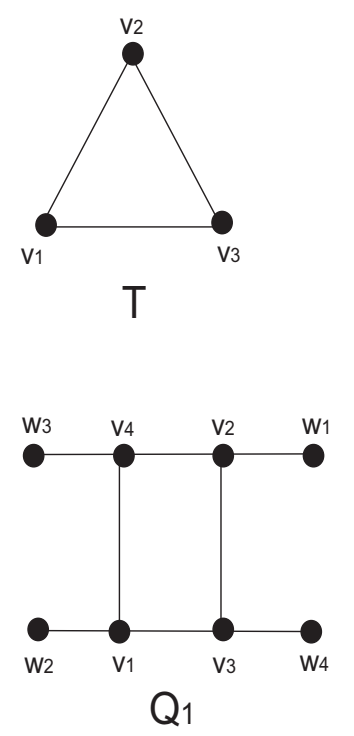
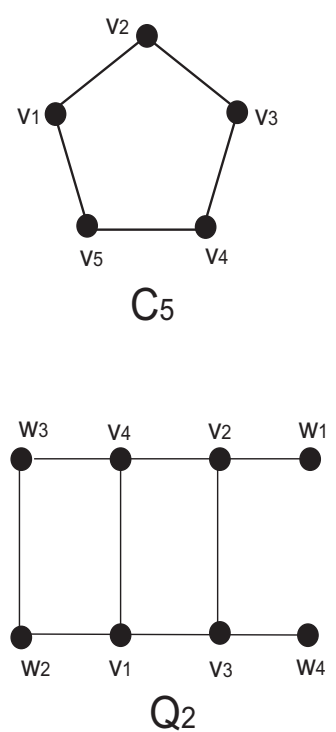
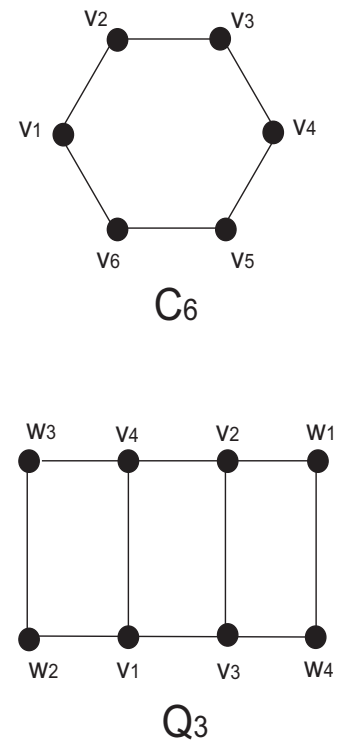

Fig. 2: A hereditary biclique-Helly graph does not contain any of these graphs as induced subgraphs.

\section{Hereditary biclique-Helly graphs}

In this section, we describe a characterization for hereditary biclique-Helly graphs. Start with the following definition.

Let $S \subseteq V(G),|S|=3$. Denote by $\mathcal{B}_{S}$ the family of bicliques of $G$, each of them containing at least two vertices of $S$. Consider the graph $G_{\mathcal{B}_{S}}$ and denote its vertex set by $S^{*} \subseteq V(G)$. The induced subgraph $G\left[S^{*}\right]$ is called the extension of $S$. Clearly, $G_{\mathcal{B}_{S}}$ is a spanning subgraph of $G\left[S^{*}\right]$. The lemma below is useful.

Lemma 2.1 [11]: Let $G$ be a graph with neither triangles nor $C_{5}$ 's. Then each of its extensions is a bipartite graph.

Next, the characterization is formulated.

Theorem 2.1 : A graph $G$ is hereditary biclique-Helly if and only if it does not contain any of the graphs of Figure 2 as induced subgraphs.

Proof: To prove that the graphs of Figure 2 are not biclique-Helly, we show a pairwise intersecting family $\mathcal{B}$ of bicliques with no common vertex, in each case. For the triangle, $\mathcal{B}=\left\{\left\{v_{1}, v_{2}\right\}\right.$, $\left.\left\{v_{1}, v_{3}\right\},\left\{v_{2}, v_{3}\right\}\right\}$ and for the $C_{5}, \mathcal{B}=\left\{\left\{v_{1}, v_{2}, v_{3}\right\},\left\{v_{3}, v_{4}, v_{5}\right\},\left\{v_{1}, v_{2}, v_{5}\right\}\right\}$. For the $C_{6}$, the family is $\mathcal{B}=\left\{\left\{v_{1}, v_{2}, v_{3}\right\},\left\{v_{3}, v_{4}, v_{5}\right\},\left\{v_{1}, v_{5}, v_{6}\right\}\right\}$. Finally, for the graphs $Q_{1}, Q_{2}$ and $Q_{3}, \mathcal{B}=\left\{\left\{v_{1}, w_{2}\right.\right.$, $\left.\left.v_{3}, v_{4}\right\},\left\{v_{2}, v_{3}, v_{4}, w_{1}\right\},\left\{v_{3}, w_{4}, v_{1}, v_{2}\right\},\left\{v_{1}, v_{2}, v_{4}, w_{3}\right\}\right\}$.

Conversely, let $G$ be a graph that does not contain any of the graphs of Figure 2 , as an induced subgraph. Suppose it is not hereditary biclique-Helly. Let $H$ be an induced subgraph that is not biclique-Helly, and $\mathcal{B}$ a non-Helly family of bicliques of $H$. We can choose $\mathcal{B}=\left\{B_{1}, \ldots, B_{k}\right\}$ as a minimal such family. 


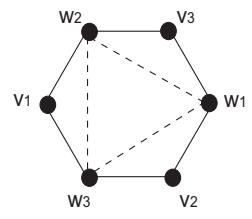

Fig. 3: $k=5$

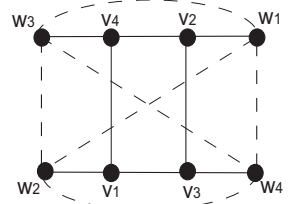

Fig. 4: $k=4$

Clearly, $k \geq 3$. As for every $i, \mathcal{B} \backslash B_{i}$ is a Helly family, there exists a vertex $v_{i}$ that belongs to $B_{j}$ and not to $B_{i}$, for all $j \neq i$. Let $S=\left\{v_{1}, \ldots, v_{k}\right\}$ be the collection of such vertices. Write $\alpha_{S}=\alpha(H[S])$.

First, we show that $\alpha_{S} \leq 2$. Assume this condition to be false and let $\left\{v_{1}, v_{2}, v_{3}\right\} \subseteq S$ be an independent set of $H$. Since $H$ has no triangles and $v_{i} \notin B_{i}$, there exists a vertex $w_{i} \in B_{i}$ adjacent to $v_{j}$ and not to $v_{i}$, for $j \neq i$ and $1 \leq i \leq 3$. See Figure 3 Then $\left\{w_{1}, w_{2}, w_{3}\right\}$ is also an independent set. In the latter situation, $\left\{v_{1}, w_{2}, v_{3}, w_{1}, v_{2}, w_{3}\right\}$ induces a $C_{6}$, which is forbidden. Consequently, indeed $\alpha_{S} \leq 2$.

In the sequel, we discuss the possible values $k$ can assume.

Let $k \geq 5$. Denote $S^{\prime}=\left\{v_{1}, v_{2}, v_{3}, v_{4}\right\}$. Clearly, $S^{\prime} \subseteq B_{5}$. For this reason and considering that $\alpha_{S} \leq 2$, we know that $S^{\prime}$ induces a $C_{4}$ in $H$. Let $v_{1} v_{3}$ and $v_{2} v_{4}$ be the non-adjacent pairs in $S^{\prime}$. Again, because $\alpha_{S} \leq 2, v_{5}$ must be adjacent to at least one vertex of $S^{\prime}$, say adjacent to $v_{1}$. Because $H$ has no triangles, $v_{5}$ can be adjacent neither to $v_{2}$, nor to $v_{4}$. However, in this situation, $\left\{v_{2}, v_{4}, v_{5}\right\}$ is an independent set of size 3, a contradiction. Consequently, $k<5$.

Next, discuss the case $k=4$. Let $S^{\prime}=\left\{v_{1}, v_{2}, v_{3}\right\} \subseteq S$. Since $S^{\prime} \subseteq B_{4}$ and $\alpha_{S} \leq 2, S^{\prime}$ induces a $P_{3}$ in $H$. Let $v_{1}$ and $v_{2}$ be the non-adjacent vertices in $S^{\prime}$. As $v_{1}, v_{2}, v_{4} \in B_{3}$ and $\alpha_{S} \leq 2$, it follows that $S$ must induce a $C_{4}$ in $H$. On the other hand, since $v_{i} \notin B_{i}$ and $v_{j} \in B_{i}$ for $j \neq i$, each $B_{i}$ has an additional vertex $w_{i} \in B_{i}, 1 \leq i \leq 4$ with the following properties: because $v_{1} \notin B_{1}$ and $H$ has no triangles, $w_{1}$ is adjacent to $v_{2}$ and not adjacent to $v_{1}, v_{3}, v_{4}$. Similarly, $w_{2}$ is adjacent to $v_{1}$, and not to $v_{2}, v_{3}, v_{4}$, and $w_{3}$ is adjacent to $v_{4}$ and not $v_{1}, v_{2}, v_{3}$, while $w_{4}$ is adjacent to $v_{3}$ and not $v_{1}, v_{2}, v_{4}$. See Figure 4

Let us examine the possible adjacencies among the $w_{i}$ 's. If $w_{1}$ and $w_{2}$ are adjacent, then $H$ contains a $C_{5}$, which is forbidden. Similarly, if $w_{3}$ and $w_{4}$ are adjacent. So, assume these pairs are not adjacent. Let $W=\left\{w_{1} w_{3}, w_{1} w_{4}, w_{2} w_{3}, w_{2} w_{4}\right\}$ be the set of the other possible pairs of $w_{i}$ 's and denote $P=$ $\left\{v_{1}, v_{2}, v_{3}, v_{4}, w_{1}, w_{2}, w_{3}, w_{4}\right\}$. If none of the pairs of $W$ is adjacent, then $P$ induces the graph $Q_{1}$. When exactly one of the pairs of $W$ is adjacent, then $P$ forms the graph $Q_{2}$. When precisely the pairs $w_{1} w_{3}$ and $w_{2} w_{4}$, or $w_{1} w_{4}$ and $w_{2} w_{3}$ are adjacent, then $P$ induces $Q_{3}$. Finally, if at least two consecutive pairs of the cyclic sequence $w_{1}, w_{4}, w_{2}, w_{3}, w_{1}$ are adjacent in $H$, then a $C_{6}$ exists. Consequently, $k=4$ is not possible.

Next, consider the case $k=3$, and let $S=\left\{v_{1}, v_{2}, v_{3}\right\}$. Denote by $\mathcal{B}_{S}$ the subset of bicliques of $H$ 


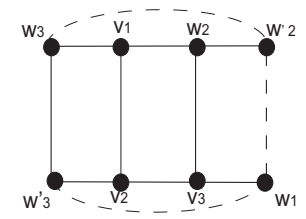

Fig. 5: $k=3$

containing at least two vertices of $S$. Then $\left\{B_{1}, B_{2}, B_{3}\right\} \subseteq \mathcal{B}_{S}$. Discuss the possibilities for $S$. Clearly, $S$ is not a triangle. Since $\alpha_{S} \leq 2, S$ is not an independent set. Suppose $S$ induces a $\overline{P_{3}}$, with $v_{1}, v_{2}$, adjacent. As $v_{2}, v_{3} \in B_{1}$, there is a vertex $w_{1} \in B_{1}$ adjacent to $v_{2}$ and $v_{3}$. Similarly, there exists $w_{2} \in B_{2}$, with $w_{2}$ adjacent to $v_{1}$ and $v_{3}$. In this case if $w_{1}, w_{2}$ are adjacent, then a triangle is formed, otherwise $\left\{v_{1}, v_{2}, w_{1}, v_{3}, w_{2}\right\}$ induces a $C_{5}$, which is forbidden.

Let us examine the remaining alternative, that is $S$ induces a $P_{3}$. By Lemma $1, H\left[S^{*}\right]$ is bipartite. Let $X \cup Y$ be a bipartition of it. Let $v_{1}, v_{3}$ be the non-adjacent pair of vertices of $S$. Let $v_{1}, v_{3} \in X$ and $v_{2} \in Y$. Because $v_{i} \notin B_{i}$ and $v_{j} \in B_{i}$, for $j \neq i$, there is a vertex $w_{i} \in B_{i}, w_{i} \neq v_{j}$, where $i, j \in\{1,3\}$, such that $w_{1} \in Y$ is adjacent to $v_{3}$ and not adjacent to $v_{1}$ and $v_{2}$, while the vertex $w_{3} \in Y$ is adjacent to $v_{1}$ and not to $v_{3}$ and $v_{2}$. Also, as $B_{2}$ is a biclique, there exists some vertex $w_{2} \in Y$ adjacent to $v_{1}$ and $v_{3}$. Because $v_{2} \notin B_{2}$, there is also a vertex $w_{2}^{\prime} \in X \cap B_{2}$ that is adjacent to $w_{2}$ and not to $v_{2}$. Since $\left\{B_{1}, B_{2}, B_{3}\right\}$ is not a Helly family, $w_{2}$ is not common to these three bicliques. Without loss of generality, assume $w_{2} \notin B_{3}$. Then there exists a vertex $w_{3}^{\prime} \in X$ that belongs to $B_{3}, w_{3}^{\prime}$ adjacent to $v_{2}$ and $w_{3}$, and not to $w_{2}$. See Figure 5 .

Let $P=\left\{v_{1}, v_{2}, v_{3}, w_{1}, w_{2}, w_{2}^{\prime}, w_{3}, w_{3}^{\prime}\right\}$ and $W=\left\{w_{1} w_{2}^{\prime}, w_{1} w_{3}^{\prime}, w_{3} w_{2}^{\prime}\right\}$. The pairs listed in $W$ are the only possible edges, and then $w_{1} w_{3}^{\prime}$ or $w_{2}^{\prime} w_{3}$ creates a $C_{6}$, and otherwise $P$ induces a $Q_{3}$ or a $Q_{2}$ depending on the presence or absence of edge $w_{1} w_{2}^{\prime}$. All cases lead to forbidden subgraphs.

Consequently, $k=3$ is not possible. However, $k \geq 3$. This contradiction completes the proof.

\section{Hereditary open and closed neighbourhood Helly graphs}

We describe below characterizations for the classes of hereditary open and closed neighbourhood-Helly graphs.

Theorem 3.1 Let $G$ be a graph. Then $G$ is hereditary open neighbourhood-Helly if and only if G contains neither $C_{6}$ nor a triangle as induced subgraphs.

Proof: Let $G$ be a hereditary open neighbourhood-Helly graph. Clearly, no subset $\left\{v_{1}, v_{2}, v_{3}\right\} \subseteq V(G)$ induces a triangle in $G$, otherwise $N\left(v_{1}\right), N\left(v_{2}\right), N\left(v_{3}\right)$ pairwise intersect, but there is no common vertex. Suppose $G$ contains a $C_{6}$. Let $v_{1}, v_{2}, v_{3}, v_{4}, v_{5}, v_{6}$ be the ordering of the vertices in this cycle. Since $N\left(v_{1}\right), N\left(v_{3}\right), N\left(v_{5}\right)$ pairwise intersect with no common vertex, we conclude that $G$ contains no $C_{6}$.

Conversely, assume the theorem is not true. Then $G$ contains an induced subgraph $H$ that is not open neighbourhood-Helly. Consider a minimal family $N=\left\{N\left(v_{1}\right), N\left(v_{2}\right), \ldots, N\left(v_{l}\right)\right\}$ of pairwise intersecting neighbourhoods with no common vertex in $H$. Then $l \geq 3$. Because of the minimality, there is a 
vertex $w_{i} \in N\left(v_{j}\right)$ precisely when $i \neq j$, for all $1 \leq i \leq l$. Since $v_{i} \notin N\left(v_{i}\right)$, we conclude that $w_{i} \neq v_{j}$, for all $i \neq j$. Moreover, we show that $w_{i} \neq v_{i}$ for all $i$. Suppose that the latter assumption is not true and let let $w_{i}=v_{i}$. Then, $v_{i}$ and $v_{j}$ are adjacent. On the other hand, since $N\left(v_{i}\right)$ and $N\left(v_{j}\right)$ intersect, there is a vertex $w$ forming a triangle with $v_{i}$ and $v_{j}$, which is forbidden by hypothesis. Consequently, $w_{i} \neq v_{j}$, for $1 \leq i, j \leq l$. We claim that $w_{1}, \ldots, w_{l}$ form an independent set in $G$. The latter is true because, if $w_{i}, w_{j}$ are adjacent, the fact that $w_{i}, w_{j} \in N\left(v_{k}\right)$, for $k \neq i, j$, implies that $w_{i}, w_{j}, v_{k}$ form a triangle of $G$, contradicting the hypothesis. Similarly, $v_{1}, \ldots, v_{l}$ also form an independent set, otherwise $v_{i} \in N\left(v_{j}\right)$ implies that $v_{i}, v_{j}, w_{k}, k \neq i, j$ are vertices of a triangle. In this situation, $w_{i}, w_{j}, w_{k}, v_{i}, v_{j}, v_{k}$ induce a $C_{6}$ in $G$, which is impossible. The proof is complete.

Next we consider the closed neighbourhood-Helly class.

Theorem 3.2 A graph $G$ is hereditary closed neighbourhood-Helly if and only if it contains neither $C_{4}$, $C_{5}, C_{6}$ nor the Hajos graph as induced subgraphs.

Proof: Suppose $G$ contains the Hajós graph $H$. The family of the closed neighbourhoods of the three vertices with degree two in $H$ is intersecting and has no common vertex. Consequently, $G$ cannot contain the Hajós graph. Suppose $G$ contains a $C_{4}$. The family of closed neighbourhoods of its four vertices violates the Helly Property. Therefore, no $C_{4}{ }^{\prime} s$ can exist. Similarly, the families of the closed neighbourhoods of three vertices in a $C_{5}$, two of them non-adjacent, and the closed neighbourhoods of the three mutually non-adjacent vertices in a $C_{6}$, are both intersecting and have no common vertex. Consequently, $G$ contains neither $C_{5}$ nor $C_{6}$.

Conversely, by hypothesis $G$ contains neither the Hajós graph, nor any of $C_{4}, C_{5}, C_{6}$ as induced subgraphs. Assume that the theorem is false and that $G$ is not a hereditary closed neighbourhood-Helly graph. Denote by $N=\left\{N\left[v_{1}\right], N\left[v_{2}\right], \ldots, N\left[v_{l}\right]\right\}$ a minimal such intersecting family of $G$ with no common vertex. Clearly, $l \geq 3$. By the minimality of $l$, there exist vertices $w_{i}$, such that $w_{i} \in N\left[v_{j}\right]$, exactly for $i \neq j$. Compare $w_{i}$ and $v_{j}$. It is clear that, $v_{i} \neq w_{i}$. Suppose $w_{i}=v_{j}$, for some $i, j$. Without loss of generality, let $w_{1}=v_{2}$. Then, $v_{1}, v_{2}$ are not adjacent, implying $v_{1} \neq w_{3}$. The latter means that $w_{1}, w_{2} \neq v_{3}$. In this situation, if $v_{1}, v_{3}$ are adjacent, the vertices $w_{1}, w_{3}, v_{1}, v_{3}$ induce a $C_{4}$, which is forbidden. Consequently, $v_{1}, v_{3}$ are not adjacent. Consider vertex $w_{2}$. It follows that when $w_{2}, w_{3}$ are adjacent, the vertices $w_{1}, w_{3}, w_{2}, v_{3}$ induce a $C_{4}$, and otherwise $w_{1}, w_{3}, v_{1}, w_{2}, v_{3}$ induce a $C_{5}$ in $G$. Hence, this alternative cannot occur. Finally, assume $w_{i} \neq v_{j}$, for all $i, j$. Since $G$ contains no $C_{6},\left\{v_{1}, v_{2}, v_{3}\right\}$ and $\left\{w_{1}, w_{2}, w_{3}\right\}$ cannot be both independent sets. Suppose $w_{2}, w_{3}$ are adjacent. Since $G$ contains neither $C_{4}$ nor $C_{5}$, we conclude that $w_{1}$ must be adjacent to both $w_{2}, w_{3}$. In this situation, if $\left\{v_{1}, v_{2}, v_{3}\right\}$ is an independent set, $w_{1}, w_{2}, w_{3}, v_{1}, v_{2}, v_{3}$ induce the Hajós graph, otherwise, they induce a $C_{4}$. In any alternative, a forbidden subgraph arises. The alternative $v_{2}, v_{3}$ to be adjacent instead of $w_{2}, w_{3}$, is similar, terminating the proof.

\section{Relations among the classes}

The hereditary classes here considered can be related by employing the characterizations formulated before. The following lemma is useful.

Lemma 4.1 [11]: Let $G$ be an open neighbourhood-Helly graph. Then $G$ has no triangles.

The corollary below relates hereditary open neighbourhood-Helly graphs to the open neighbourhoodHelly graphs. It is a consequence of Theorem 3.1 and Lemma 4.1 
Corollary 4.1 A graph is hereditary open neighbourhood-Helly if and only if it is open neighbourhoodHelly and has no $C_{6}$.

The next lemma relates hereditary closed neighbourhood-Helly graphs to the closed neighbourhoodHelly graphs.

Lemma 4.2 Let $G$ be a closed neighbourhood-Helly graph, with no triangles. Then $G$ is hereditary closed neighbourhood-Helly.

Proof: To prove that $G$ is hereditary closed neighbourhood-Helly, we show that $G$ does not contain contain $C_{4}, C_{5}$ or $C_{6}$ as induced subgraphs. Assume the contrary, that is, $G$ contains a $C_{4}$ as an induced subgraph. Consider the intersecting family $\left\{N\left[v_{i}\right]\right\}, 1 \leq i \leq 4$. By hypothesis, it has a common intersection which implies that a triangle is formed, a contradiction. Then, $C_{4}$ is not an induced subgraph of $G$. Similarly, suppose $G$ contains a $C_{5}$. The family of closed neighbourhoods of the five vertices of the $C_{5}$ intersects in a vertex, forming a triangle, again a contradiction. Finally, consider the closed neighbourhoods of the three mutually non-adjacent vertices of a $C_{6}$. As they have a common vertex, there exists an induced $C_{4}$ or a triangle, a contradiction.

As a consequence of Lemma 4.2 we obtain the following relation between closed neighbourhood-Helly graphs and other hereditary Helly classes.

Corollary 4.2 If $G$ is closed neighbourhood-Helly with no triangles, then it is hereditary biclique-Helly, hereditary open neighbourhood-Helly and hereditary closed neighbourhood-Helly.

Finally we can also conclude:

Corollary 4.3 Let $G$ be a graph with girth at least 7. Then $G$ is hereditary biclique-Helly, hereditary open neighbourhood-Helly and hereditary closed neighbourhood-Helly.

\section{Acknowledgements}

To Pavol Hell, for the helpful discussions on the subject.

\section{References}

[1] H.-J. Bandelt and V. Chepoi. "Metric graph theory and geometry: a survey", in J. E. Goodman, J. Pach and R. Pollack, eds., Contemporary Mathematics, Proceedings of the Joint Summer Research Conference on Discrete and Computational Geometry, 2006, to appear.

[2] H.-J. Bandelt, M. Farber and P. Hell. "Absolute reflexive retracts and absolute bipartite graphs", Discrete Applied Mathematics 44: 9-20, 1993.

[3] H.-J. Bandelt and E. Prisner. "Clique graphs and Helly graphs", Journal of Combinatorial Theory B 51: 34-45, 1991.

[4] H.-J. Bandelt and E. Pesch. "Dismantling absolute retracts of reflexive graphs", European Journal of Combinatorics 10: 211-220, 1989.

[5] C. Berge. "Hypergraphs", North Holland Mathematical Library, vol. 45, Elsevier Science Publishers B.V., Amsterdam, 1989. 
[6] A. Bondy, G. Durán, M. Lin and J. L. Szwarcfiter. "Self-clique graphs and matrix permutations", Journal of Graph Theory 44: 178-192, 2003.

[7] A. Brandstädt, V. Le and J. Spinrad. "Graph Classes. A survey”, SIAM Monographs on Discrete Mathematics and Applications, 1999.

[8] P. Burzyn, F. Bonomo and G. Durán. "NP-completeness results for edge modification problems", Discrete Applied Mathematics 154: 1824-1844, 2006.

[9] F. F. Dragan. "Centers of Graphs and the Helly property", PhD thesis, Moldova State University, Chisinau, Moldova, 1989. In russian.

[10] F. Escalante. Über iterierte Clique-Graphen, Abhändlungen der Mathematischen Seminar der Universität Hamburg 39: 59-68, 1973.

[11] M. Groshaus and J. L. Szwarcfiter. "Biclique-Helly graphs", Graphs and Combinatorics, to appear. DOI 10.1007/s00373-007-0756-6.

[12] B. C. Hamelink. "A partial characterization of clique graphs", Journal of Combinatorial Theory 5: 192-197, 1968.

[13] P. Hell, Personal Communication, 2003.

[14] F. Larrión, V. Neumann-Lara, M. A. Pizaña and T. D. Porter. "A hierarchy of self-clique graphs", Discrete Mathematics 282: 193-208, 2004.

[15] H. Müller. "On edge perfectness and classes of bipartite graphs", Discrete Mathematics 149: 159$187,1996$.

[16] R. Peeters. "The maximum edge biclique problem is NP-complete", Discrete Applied Mathematics 131: 651-654, 2003.

[17] E. Prisner. "Bicliques in graphs, I: Bounds on their number", Combinatorica 20: 109-117, 2000.

[18] E. Prisner. "Hereditary clique-Helly graphs", Journal of Combinatorial Mathematics and Combinatorial Computing 14: 216-220, 1993.

[19] F. S. Roberts and J. H. Spencer. "A characterization of clique graphs", Journal of Combinatorial Theory B 10: 102-108, 1971.

[20] Z. Tuza. "Covering of graphs by complete bipartite subgraphs: complexity of 0-1 matrices", Combinatorica 4: 111-116, 1984. 